\title{
Effects of Atomoxetine on Levels of Monoamines and Related Substances in Discrete Brain Regions in Mice Intermittently Deprived of Rapid Eye Movement Sleep
}

\author{
Fukie Nitjima, ${ }^{*}, a$ Hiroko Saito,${ }^{b}$ Shigeo MuraI,${ }^{b}$ Yuichiro Arai,${ }^{c}$ Osamu Nakagawasai, ${ }^{a}$ \\ Koichi TAN-No, ${ }^{a}$ Hiromi Watanabe, ${ }^{a}$ Hajime Hiraga,,${ }^{a}$ and Takeshi TADANo ${ }^{a}$ \\ ${ }^{a}$ Department of Pharmacology, Tohoku Pharmaceutical University; 4-4-1 Komatsushima, Aoba-ku, Sendai 981-8558, \\ Japan: ${ }^{b}$ Laboratory of Pharmacology, Faculty of Pharmaceutical Science, Aomori University; 2-3-1 Koubata, Aomori \\ 030-0943, Japan: and ${ }^{c}$ Department of Judotherapy, Faculty of Health Sciences, Tokyo Ariake University of Medical and \\ Health Sciences; 2-9-1 Ariake, Koto-ku, Tokyo 135-0063, Japan.
}

Received September 18, 2009; accepted December 22, 2009

An imbalance between noradrenergic and dopaminergic systems is implicated in hyperactivity disorders, such as attention deficit/hyperactivity disorder (ADHD). We previously showed that the explosive jumping behavior elicited by intermittent rapid eye movement sleep deprivation (REMSD) may serve as a useful model of ADHD (see [Biogenic Amines, 20, 99-111]). Here, we investigated whether intermittent REMSD might cause changes in monoamine turnover in the mouse forebrain. Our main findings were as follows. Intermittent REMSD led to a significant decrease in dopamine turnover and a significant increase in noradrenaline turnover in the frontal cortex. The latter effect, but not the former, was attenuated by atomoxetine, which is used clinically to treat ADHD symptoms. These results suggest (a) that intermittent REMSD induces hypernoradrenergic and hypodopaminergic states within the frontal cortex, and (b) that the therapeutic effects of atomoxetine may include an inhibition of this hypernoradrenergic state.

Key words sleep deprivation; attention deficit/hyperactivity disorder; atomoxetine; noradrenaline; dopamine; frontal cortex

Sleep deprivation represents a common type of stress that can, when extreme, lead to psychological and physiological damage, and even death, in experimental animals. ${ }^{1)}$ After a period of intermittent rapid eye movement sleep deprivation (REMSD) (72-96h), rats stay awake for approximately $30 \mathrm{~min}$, and during this period display hyperactivity, irritability, aggressiveness, and hypersexuality. ${ }^{2)}$ Recently, we showed in mice that 5 days' intermittent REMSD $(20 \mathrm{~h} / \mathrm{d})$ results in explosive jumping behavior. This behavior was antagonized dose-dependently and significantly by treatment with atomoxetine $(0.41-0.55 \mathrm{mg} / \mathrm{kg}$, subcutaneously (s.c.)), which is used clinically to treat the symptoms of attention deficit/hyperactivity disorder (ADHD), ${ }^{3)}$ raising the possibility such jumping behavior may serve as a useful animal model of ADHD. Moreover, there may be a positive association between this animal model and a hyperfunctional status of noradrenergic systems since the firing of noradrenergic neurons in locus ceruleus (LC) continuous during rapid eye movement (REM) sleep deprivation. ${ }^{4-6)}$

ADHD is a childhood psychiatric disorder characterized by the presence of symptoms in three domains: inattention, impulsivity, and motor overactivity. ${ }^{7)}$ It has been suggested that these symptoms may be due to a combination of alerting deficits and executive-control deficits involving the prefrontal cortex. ${ }^{8,9)}$ Although existing studies contain several inconsistencies, on balance the evidence supports dysfunctions of the fronto-striatal neuronal circuits regulated by dopaminergic and noradrenergic afferents, with resultant executive deficits in cognitive functioning. ${ }^{7-10}$ )

The stimulants amphetamine and methylphenidate, which are indirect agonist that increase extracellular monoamine concentration by inhibiting reuptake and/or promoting release, are the primary treatment for ADHD. ${ }^{11)}$ The efficacy of stimulants suggests that dopaminergic and/or noradrenergic dysregulation contribute to the expression of ADHD, while diversity in psychostimulant response suggests that the ADHD is not attributable to any single pathophysiologic mechanism. In the absence of specific therapeutic targets, the treatment strategy progresses from stimulant to non-stimulant drugs. ${ }^{12)}$

Atomoxetine is a non-stimulant drug and a selective inhibitor of the presynaptic noradrenaline (NA)-reuptake transporter (NAT). Indeed, it has an approximately 300 -fold selectivity for NAT over the dopamine (DA)-uptake transporter (DAT), a finding that has increased interest in the role of NAT and noradrenergic systems in ADHD. ${ }^{13)}$ In the frontal cortex, NAT density is higher than DAT density, ${ }^{14-16)}$ and NAT blockade by atomoxetine is thought to block sequestration of DA into NA neurons. ${ }^{13)}$ Moreover, NAT is poorly expressed in the striatum, in contrast to its robust expression within the frontal cortex. ${ }^{13)}$ The above findings suggest that the therapeutic effects of drugs that block NAT are mediated by increasing both NA and DA in noradrenergic terminal areas such as the frontal cortex (versus the striatum).

The aims of the present study, on mice, were to investigate (a) whether intermittent REMSD induces changes in the levels of monoamine-related substances [noradrenaline (NA), methoxyhydroxyphenylglycol (MHPG, a NA metabolite), dopamine (DA), dihydroxyphenylacetic acid (DOPAC, a DA metabolite), and homovanillic acid (HVA, a DA metabolite)] in the frontal cortex and striatum, and (b) the effects of atomoxetine treatment on such changes.

\section{MATERIALS AND METHODS}

Animals Male ddY-strain mice (Japan SLC, Hamamatsu, Japan) weighing $19-21 \mathrm{~g}$ were used in all experiments. They were given standard food and tap water ad libi- 
tum in an air-conditioned room at $23 \pm 1{ }^{\circ} \mathrm{C}$ and $55 \pm 5 \%$ relative humidity with a standard $12 \mathrm{~h}$ light $/ 12 \mathrm{~h}$ dark cycle (lights on 08:00 a.m.)

Drugs Atomoxetine (Sigma Chemical Co., St. Louis, MO, U.S.A.) was dissolved in saline and administered s.c. All injections were in a volume of $0.1 \mathrm{ml} / 10 \mathrm{~g}$ body weight.

Intermittent REMSD Treatment Mice were deprived of REM sleep intermittently by the small-platform method, as described by Niijima et $a l^{3)}$ In brief, a small platform ( $4.5 \mathrm{~cm}$ high, $1.8 \mathrm{~cm}$ diameter) fixed to the center of a plastic cage $(22.0 \mathrm{~cm} \times 15.0 \mathrm{~cm} \times 12.5 \mathrm{~cm})$ was surrounded with water $3.5 \mathrm{~cm}$ deep. Mice were placed individually on the platform within the plastic cage, and were housed therein for $20 \mathrm{~h}$ once a day with food and water being supplied ad libitum. After such REMSD treatment for $20 \mathrm{~h}$, each mouse was placed individually in a normal plastic cage and allowed $4 \mathrm{~h}$ of rest for that day. This schedule was repeated for $5 \mathrm{~d}$. Tankcontrol (TC) animals were submitted to the same procedure, except that the platforms were $10 \mathrm{~cm}$ in diameter. Cage-control (CC) animals were housed in groups in plastic cages $(32.0 \mathrm{~cm} \times 21.0 \mathrm{~cm} \times 12.5 \mathrm{~cm})$.

Determination of Neurotransmitter Concentrations The determination of neurotransmitter concentrations was carried out essentially by the method described by Saito et al. ${ }^{17)}$ Briefly, immediately after the end of the intermittent REMSD treatment, mice were injected with atomoxetine or saline and placed individually in the home cage. Thirty five minutes later, each mouse was killed by microwave irradiation (4kW, 1.2 s; Model TMW-6402A; Toshiba, Tokyo, Japan). Brains were removed and two brain regions (frontal cortex and striatum) were dissected out, as previously described by Glowinski and Iversen. ${ }^{18)}$ The dissected tissues were quickly frozen on dry ice, weighed, and stored in a $1.5 \mathrm{ml}$ microtube at $-80^{\circ} \mathrm{C}$ until extraction. Tissues were then homogenized for $10 \mathrm{~s}$ using an ultrasonic cell disruptor (Model 200; 60 W, 50\% pulsed power; Branson, Danbury, CT, U.S.A.). This was done in ice-cold $0.05 \mathrm{M}$ perchloric acid (PCA) solution (400 $\mu \mathrm{l}$ for striatum, $200 \mu \mathrm{l}$ for frontal cortex). Homogenates were centrifuged at $12000 \times \boldsymbol{g}$ for $15 \mathrm{~min}$ at $4{ }^{\circ} \mathrm{C}$, and the clear supernatants were decanted and filtered through a $0.45-\mu \mathrm{m}$ filter (Type $\mathrm{HV}$; Nihon Millipore, Yonezawa, Japan), then stored at $-80{ }^{\circ} \mathrm{C}$ until assayed. The filtrate was used for quantification of NA (Wako Chemicals, Osaka, Japan), MHPG (Sigma, St. Louis, MO, U.S.A.), DA (Kanto Chemicals, Tokyo, Japan), DOPAC (Aldrich, Milwaukee, WI, U.S.A.), and HVA (Sigma, St. Louis, MO, U.S.A.) by HPLC coupled with coulometric detection. The HPLC system consisted of a solvent delivery pump (Model EP-10S; Eicom, Kyoto, Japan), an autosampler (Model AS4000; Hitachi, Tokyo, Japan), and an analytical column (C18 Ultrasphere; $75 \mathrm{~mm} \times 4.6 \mathrm{~mm}$ i.d., $3-\mu \mathrm{m}$ particle size; Beckman, San Ramon, CA, U.S.A.) protected by a guard column (Eicom prepak; $5 \mathrm{~mm} \times 4.6 \mathrm{~mm}$ i.d., $7-\mu \mathrm{m}$ particle size, Eicom, Kyoto, Japan). The ESA model 5100A Coulochem electrochemical detection system (ESA Inc., Bedford, MA, U.S.A.) consisted of a model 5021 conditioning cell (detector setting, $+0.4 \mathrm{~V}$ ) followed by a model 5011 dual electrode analytical cell (cell $1,+0.02 \mathrm{~V}$; cell $2,-0.3 \mathrm{~V}$ ). These oxidative and reductive potentials were set on the basis of the current-voltage curves published by Kurata et al. ${ }^{19)}$ and Takeda et $a l .^{20)}$ The output signal from the final electrode was ampli- fied by the $5100 \mathrm{~A}$ controller and relayed to an integrator (Model C-R6A, Shimadzu, Kyoto, Japan). The temperature of the analytical and guard columns, which was controlled by a column jacket connected to a thermostatic water-bath (Model UC-65; Tokyo Rika Kikai, Tokyo, Japan), was kept at $35^{\circ} \mathrm{C}$. The mobile phase was $0.02 \mathrm{M}$ sodium acetate/ $0.0125 \mathrm{M}$ citric acid buffer, $\mathrm{pH} 3.7$, containing $8 \%(\mathrm{v} / \mathrm{v})$ methanol, $0.042 \%$ heptanesulfonic acid (C7), and $0.1 \mathrm{~mm}$ $\mathrm{Na}_{2}$ ethylenediaminetetraacetic acid (EDTA). The buffer solution was filtered through a $0.45-\mu \mathrm{m}$ membrane filter and degassed. The flow-rate was set to $2.4 \mathrm{ml} / \mathrm{min}$, which yielded a pressure of $190 \mathrm{~kg} / \mathrm{cm}^{2}$. The mobile phase was recycled and replaced when the baseline noise of the detector output reached an unacceptable level.

Statistical Analyses Results are expressed as means S.E.M. Comparisons were made by one-way analysis of variance combined with Scheffe's test or Dunnet's test. $p<0.05$ represented a significant difference.

\section{RESULTS}

Influence of Intermittent REMSD over Concentrations of Monoamines and Their Metabolites in Frontal Cortex and Striatum The concentrations of NA and its metabolites in the frontal cortex and striatum are given for the various groups in Table 1. Five days' intermittent REMSD significantly elevated the MHPG concentrations in the frontal cortex (versus both the TC-saline group and the CC-saline group) and striatum (versus the $\mathrm{CC}$-saline group). The concentration of NA in the striatum was significantly higher in the REMSD-saline and TC-saline groups than in the CCsaline one. The NA level in the frontal cortex of REMSD mice tended (non-significantly) to be lower than that in TC mice, but higher than that in CC mice (by $11 \%$ and $12 \%$, respectively). As shown in Table 2, the DA level in the frontal cortex of REMSD mice tended (non-significantly) to be higher than that in $\mathrm{CC}$ mice. The effects of intermittent REMSD on the MHPG/NA ratio in the frontal cortex and striatum are depicted in Fig. 1. The MHPG/NA ratio in the frontal cortex was significantly higher in the REMSD group than in either the TC-saline or CC-saline groups. In contrast,

Table 1. Effects of Intermittent REMSD and Atomoxetine Treatment on NA and MHPG Concentrations in Mouse Brain Regions

\begin{tabular}{clll}
\hline \hline \multirow{2}{*}{ Region } & \multicolumn{1}{c}{ Group } & NA & MHPG \\
\hline \multirow{2}{*}{ Frontal cortex } & CC-saline & $238 \pm 11$ & $26 \pm 1$ \\
& TC-saline & $294 \pm 14$ & $30 \pm 1$ \\
& REMSD-saline & $267 \pm 11$ & $40 \pm 2 * *$,\# \\
& CC-atomoxetine $(0.55)$ & $259 \pm 15$ & $21 \pm 1$ \\
& TC-atomoxetine $(0.55)$ & $287 \pm 10$ & $24 \pm 1$ \\
& REMSD-atomoxetine (0.55) & $273 \pm 9$ & $30 \pm 1^{\$ \$}$ \\
& CC-saline & $144 \pm 5$ & $39 \pm 2$ \\
& TC-saline & $223 \pm 10 * *$ & $47 \pm 1$ \\
& REMSD-saline & $234 \pm 14 * *$ & $54 \pm 3 * *$ \\
& CC-atomoxetine $(0.55)$ & $161 \pm 9$ & $29 \pm 1 *$ \\
& TC-atomoxetine $(0.55)$ & $176 \pm 13$ & $31 \pm 2$ \\
& REMSD-atomoxetine $(0.55)$ & $217 \pm 13$ & $37 \pm 2^{\$ \$}$ \\
\end{tabular}

Mice were treated once with saline or atomoxetine $(0.55 \mathrm{mg} / \mathrm{kg}$, s.c. $)$ immediately after the end of the 5-d REMSD period, then sacrificed $35 \mathrm{~min}$ later. Concentrations are expressed as ng per $\mathrm{g}$ of fresh-weight brain tissue. Data are expressed as mean \pm S.E.M. $(n=6-8) . * p<0.05 ; * * p<0.01 v s$. saline-treated CC. \# $p<0.01 ; v s$. saline-treated TC. $\$ \$ p<0.01 v s$. saline-treated REMSD (Scheffe's test). 
Table 2. Effects of Intermittent REMSD and Atomoxetine Treatment on DA, DOPAC, and HVA Concentrations in Mouse Brain Regions

\begin{tabular}{clccc}
\hline \hline \multicolumn{1}{c}{ Region } & \multicolumn{1}{c}{ Group } & DA & DOPAC & HVA \\
\hline \multirow{2}{*}{ Frontal cortex } & CC-saline & $52 \pm 6$ & $28 \pm 1$ & $72 \pm 4$ \\
& TC-saline & $78 \pm 7$ & $32 \pm 2$ & $78 \pm 5$ \\
& REMSD-saline & $101 \pm 23$ & $35 \pm 5$ & $74 \pm 6$ \\
& CC-atomoxetine (0.55) & $63 \pm 8$ & $29 \pm 2$ & $71 \pm 3$ \\
& TC-atomoxetine (0.55) & $92 \pm 26$ & $30 \pm 3$ & $72 \pm 10$ \\
& REMSD-atomoxetine $(0.55)$ & $80 \pm 14$ & $27 \pm 3$ & $69 \pm 5$ \\
Striatum & CC-saline & $12347 \pm 491$ & $829 \pm 42$ & $1594 \pm 64$ \\
& TC-saline & $13232 \pm 546$ & $1005 \pm 54$ & $1757 \pm 46$ \\
& REMSD-saline & $9904 \pm 497 *$ & $701 \pm 30$ & $1243 \pm 65^{*}$ \\
& CC-atomoxetine (0.55) & $12339 \pm 597$ & $820 \pm 50$ & $1415 \pm 44$ \\
& TC-atomoxetine $(0.55)$ & $11662 \pm 92$ & $809 \pm 32^{\$ \$}$ & $1625 \pm 77$ \\
& REMSD-atomoxetine $(0.55)$ & & &
\end{tabular}

Mice were treated once with saline or atomoxetine $(0.55 \mathrm{mg} / \mathrm{kg}$, s.c. $)$ immediately after the end of the 5-d REMSD period, then sacrificed $35 \mathrm{~min}$ later. Concentrations are expressed as ng per $\mathrm{g}$ of fresh-weight brain tissue. Data are expressed as mean \pm S.E.M. $(n=6-8) . * p<0.05 ; \quad v s$. saline-treated CC. $\$ p<0.01$ vs. saline-treated REMSD (Scheffe's test).

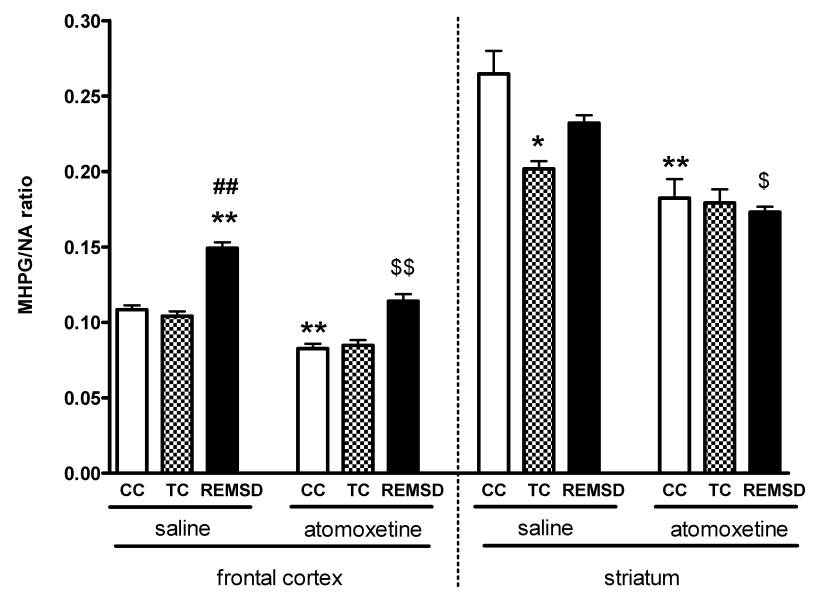

Fig. 1. Effects of Atomoxetine Treatment on the Frontal Cortex and Striatum MHPG/NA Ratio in CC, TC, and REMSD Mice

Open column, CC mice; checked column, TC mice; solid column, REMSD mice. Data are expressed as mean \pm S.E.M. $(n=6-8) . * p<0.05 ; * * p<0.01 v s$. saline-treated CC. ${ }^{\#} p<0.01 ; v s$. saline-treated TC. $\$ p<0.05 ; \$ \$ p<0.01$ vs. saline-treated REMSD (Scheffe's test).

the striatal MHPG/NA ratio was significantly lower in the TC-saline groups than in the CC-saline one. The MHPG/NA ratio in the striatum of REMSD mice tended (non-significantly) to be lower than that in $\mathrm{CC}$ mice, but higher than that in TC mice. As shown in Figs. 2 and 3, the DOPAC/DA and HVA/DA ratios in the frontal cortex were each significantly lower in the REMSD-saline group than in the CC-saline group. In the striatum, there were no significant differences in DOPAC/DA or HVA/DA ratios between REMSD mice and either CC-saline or TC-saline mice.

Effects of Atomoxetine on the Changes Induced in the Concentrations of Monoamines and Their Metabolites in Frontal Cortex and Striatum by Intermittent REM-Sleep Deprivation We previously demonstrated that atomoxetine treatment at a dose of $0.55 \mathrm{mg} / \mathrm{kg}$ (s.c.) significantly reduces the jumping behavior induced in mice by intermittent REMSD (Niijima et al. $^{3)}$ ). In CC mice: both (a) the MHPG/ NA ratio in the frontal cortex and (b) the MHPG content, MHPG/NA ratio, DA, and HVA levels in the striatum were significantly lower in the atomoxetine $(0.55 \mathrm{mg} / \mathrm{kg})$-treated

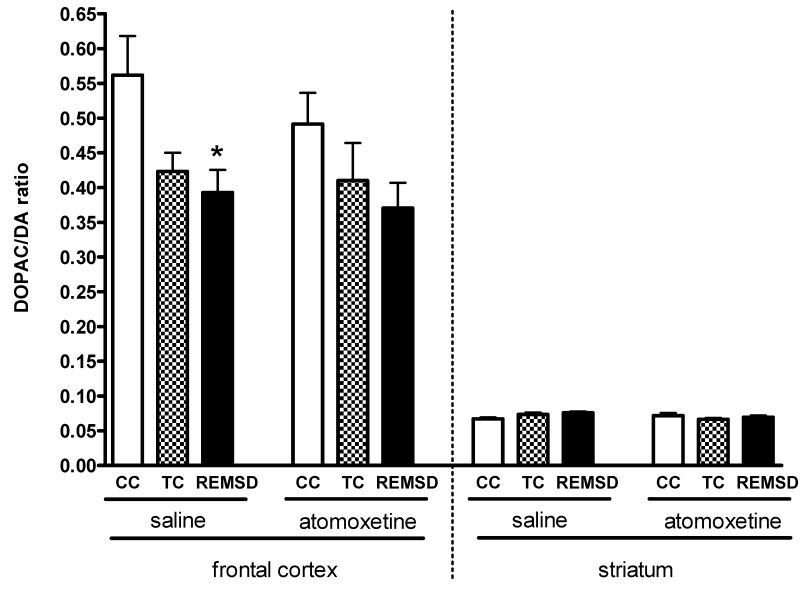

Fig. 2. Effects of Atomoxetine Treatment on the Frontal Cortex and Striatum DOPAC/DA Ratio in CC, TC, and REMSD Mice

Open column, CC mice; checked column, TC mice; solid column, REMSD mice. Data expressed as mean \pm S.E.M. $(n=6-8) . * p<0.05 ; v s$. saline-treated CC (Dunnett's test).

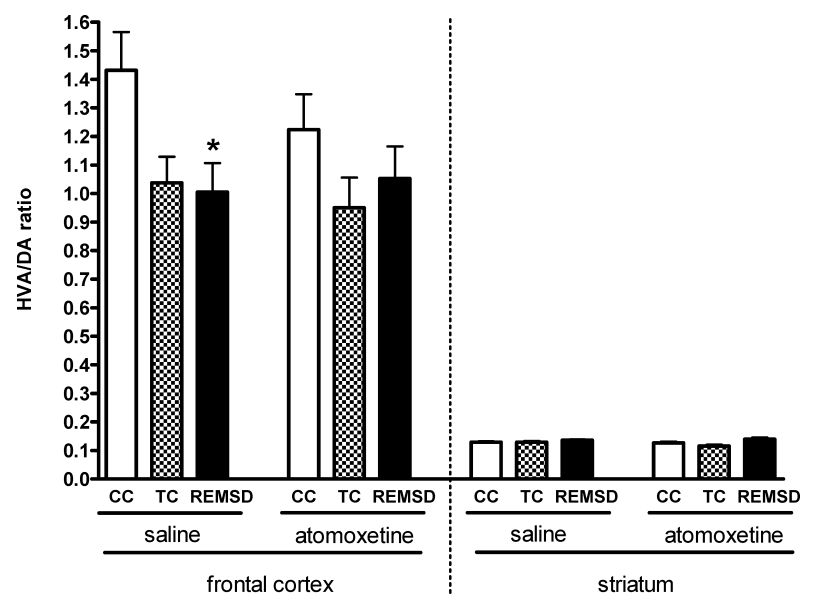

Fig. 3. Effects of Atomoxetine Treatment on the Frontal Cortex and Striatum HVA/DA Ratio in CC, TC, and REMSD Mice

Open column, CC mice; checked column, TC mice; solid column, REMSD mice. Data are expressed as mean \pm S.E.M. $(n=6-8) . * p<0.05 ; v s$. saline-treated CC (Dunnett's test).

group than in their saline-treated controls (Tables 1, 2 and Fig. 1). Next, we examined the effects of atomoxetine on those variables that were altered by REMSD. In the frontal cortex of REMSD mice, the MHPG content and MHPG/NA ratio (but not the DOPAC/DA ratio or HVA/DA ratio) were significantly lower in the atomoxetine $(0.55 \mathrm{mg} / \mathrm{kg})$-treated group than in their saline-treated controls (Table 1 and Figs. $1-3)$. In the striatum of REMSD mice, the MHPG content, and was significantly lower in the atomoxetine $(0.55 \mathrm{mg} / \mathrm{kg})-$ treated group than in their saline-treated controls (Tables 1,2 and Fig. 1).

\section{DISCUSSION}

Statistical analysis of the present neurochemical data revealed significant effects of intermittent REMSD on (a) the MHPG concentration, MHPG/NA ratio, DOPAC/DA ratio, and HVA/DA ratio in the frontal cortex, and (b) the NA and MHPG concentrations in the striatum (in each case, versus $\mathrm{CC}$ mice). The factors responsible for these alterations in 
monoamine levels in the frontal cortex and striatum cannot be determined from the present study. Previous studies have shown that a certain amount of stress is associated with the introduction of rats into a water-tank environment, regardless of pedestal size. ${ }^{21,22)}$ In the present study, we tried to exclude this confounding factor by including a stress-control group (viz. the TC group). It is important to emphasize that the nonspecific stress associated with the methodology employed here for "REM-sleep deprivation" presumably had robust effects on neurotransmission within the brain that could have masked some of the specific effects of REMSD. However, in the present study the differences detected between the REMSD mice and the TC and CC groups (in the levels of MHPG and MHPG/NA ratio in the frontal cortex) can probably be attributed to prolonged, intermittent REM sleep deprivation and not to a continuous deprivation of sleep per se.

In rats, the LC consists principally of NA-containing neurons that project to many brain regions. ${ }^{23)}$ The discharge activity of these neurons is highest during waking, decreases during slow-wave sleep, and is virtually absent during REM sleep. ${ }^{4-6)}$ In the absence of REM sleep, these neurons fire continuously, resulting in reduced receptor sensitivity ${ }^{24,25)}$ and changes associated with NA synthesis and metabolism. ${ }^{26,27)}$ Actually, the turnover and metabolism of NA are elevated both during and after REMSD. ${ }^{26-28)}$ The activity of tyrosine hydroxylase $(\mathrm{TH})$, the rate-limiting enzyme in catecholamine synthesis, is high in the whole brain after $96 \mathrm{~h}$ of REMSD, ${ }^{29)}$ and some increases in TH mRNA and/or NAT mRNA have been noted in the LC in rats exposed to $3 \mathrm{~d}$ of REMSD. ${ }^{26,30)}$ Thus, there are a substantial number of reports indicating that REMSD induces alterations in the levels of monoamines and their metabolites in different functional regions of the rat brain. The data presented here demonstrate both that the MHPG level and the MHPG/NA ratio are elevated in the frontal cortex of the intermittent REM sleep-deprived mouse, consistent with there being an enhanced NA turnover related to the hypernoradrenergic state expected to be caused by a continuous firing of LC neurons.

In the present biochemical experiment, the NA concentration and MHPG/NA ratio in the striatum were increased and decreased, respectively, in the TC mice (versus CC mice). The factors responsible for these changes in the TC mice cannot be determined from the present study. One possible mechanism may depend on the close link between activation of the hypothalamic-pituitary-adrenal axis by stress and stimulation of various neurotransmitter systems. ${ }^{30-32)}$ Previously it was shown that serum corticosteron levels are high in animals placed on small and large platform. ${ }^{26)}$ Therefore, increase in NA concentration and the decrease in MHPG/NA ratio in the striatum in the TC group might be a neurochemical correlate of the emotional arousal produced in our mice by the changes they experienced in environmental stimuli, irrespective of their aversive (REMSD) or nonaversive (TC) nature.

Dopamine transmission is traditionally associated with motor function. Brock et al. ${ }^{21)}$ reported that 4 days' REMSD in rats is associated with a significant increase in the density of DA receptors in the frontal cortex, indicating that DA activity may be decreased in this region. The dopaminergic innervation of the frontal cortex appears to have an inhibitory influence on the subcortical dopaminergic neuronal systems since reductions in prefrontal dopaminergic activity result in an increased activity or enhanced sensitivity of the subcortical systems. ${ }^{33-37)}$ In fact, the present results showed significant decrease in both the DOPAC/DA ratio and HVA/DA ratio in the frontal cortex, and an increase tendency of the DOPAC concentration in the striatum in the REMSD group (versus the CC group). Thus, a hypofunctional status of the dopaminergic systems in the prefrontal cortex of the REMSD group may be associated with an increased activity of the relevant subcortical structures, and we speculate that such a mechanism may be involved in the development of locomotor sensitization (e.g., jumping behavior).

Several lines of research suggest that ADHD symptoms may be due to an imbalance between noradrenergic and dopaminergic systems within the frontal cortex, with inhibitory dopaminergic activity being decreased and noradrenergic activity increased relative to control. ${ }^{9)}$ Our results show that significant decrease in DA turnover in the frontal cortex in the REMSD group (versus the CC), and significant increase in NA turnover in the frontal cortex in the REMSD group (versus both the $\mathrm{TC}$-saline group and the $\mathrm{CC}$-saline group). These findings may support our assumption that abnormalities in catecholaminergic regulation, especially the existence of hypernoradrenergic and/or hypodopaminergic states, are implicated in the jumping behavior induced by intermittent REMSD treatment (as a model of ADHD).

The present data indicate that in our $\mathrm{CC}$ mice, atomoxetine decreased MHPG/NA in the frontal cortex, and MHPG, MHPG/NA, DA, and HVA in the striatum. Swanson et al. ${ }^{38)}$ noted that administration of the $\alpha 2$-adrenergic antagonist idazoxan after atomoxetine resulted in an increase in prefrontal cortical NA efflux that was greater than that seen with either compound alone, indicating an attenuating effect of adrenergic autoreceptors on NA efflux. Further, a dual-probe microdialysis study demonstrated that in rats, the NA-reuptake blocker desipramine inhibits NA reuptake at the somatodendritic and nerve terminal levels in noradrenergic cells. ${ }^{39)}$ An increase in NA dialysate in the LC inhibited noradrenergic activity, which would to some extent counteract the effects of desipramine on cortical areas, and modulation of cortical NA release by an action of desipramine the somatodendritic level was found to be mediated through $\alpha 2$-adrenoceptors located within the LC. ${ }^{39)}$ In vivo microdialysis studies in anesthetized rats have also shown that after extensive denervation of the LC, there is a significant reduction in DA overflow within the striatum. ${ }^{40,41)}$ These findings suggest that the differences we detected between the saline-treated CC group and the atomoxetine-treated $\mathrm{CC}$ group (see above) may be related to stimulation of the $\alpha 2$-receptor by increased NA levels in the frontal cortex and LC after atomoxetine treatment.

The increases in MHPG in the frontal cortex and the striatum and MHPG/NA ratio in the frontal cortex induced by intermittent REMSD in our mice were decreased by atomoxetine. Basheer et al. $^{30)}$ showed that REMSD resulted in increased levels of TH mRNA and NAT mRNA in LC, indicating that REMSD might be due to increased activity of the LC neurons resulting in enhanced synthesis and release of NA at synapse which in turn results in increased expression of the transporter. Further, Asakura et al. $^{42)}$ found that REMSD enhances the sensitivity of the $\alpha 2$-adrenoceptor. These findings 
suggest that stimulation of the $\alpha 2$-receptor by the increased NA level present after atomoxetine treatment may be causally related to this drug's attenuation of the hyperadrenergic state shown to be induced by intermittent REMSD treatment in our study.

In conclusion, we found that 5 days' intermittent REMSD treatment in mice induced hypernoradrenergic and hypodopaminergic states within the frontal cortex. These results are in line with hypotheses regarding the mechanisms underlying ADHD. In addition, the observed inhibitory effect of atomoxetine on the hypernoradrenergic state may support our previous finding of an inhibitory effect of this drug on the jumping behavior induced by intermittent REMSD treatment.

\section{REFERENCES}

1) Rechtschaffen A., Bergmann B. M., Sleep, 25, 18-24 (2002).

2) Fadda P., Martellotta M. C., Gessa G. L., Fratta W., Prog. Neuropsychopharmacol. Biol. Psychiatry, 17, 269-278 (1993).

3) Niijima F., Nakagawasai O., Tan-No K., Tadano T., Biogenic Amines, 20, 99-111 (2006).

4) Aston-Jones G., Bloom F. E., J. Neurosci., 1, 876-886 (1981).

5) Foote S. L., Berridge C. W., Adams L. M., Pineda J. A., Prog. Brain Res., 88, 521-532 (1991).

6) Hobson J. A., McCarley R. W., Wyzinski P. W., Science, 189, 55-58 (1975).

7) Himelstein J., Newcorn J. H., Halperin J. M., Front Biosci., 5, D461D478 (2000).

8) Arnsten A. F., Li B. M., Biol. Psychiatry, 57, 1377-1384 (2005).

9) Russell V. A., Behav. Brain Res., 130, 191-196 (2002).

10) Teicher M. H., Anderson C. M., Polcari A., Glod C. A., Maas L. C., Renshaw P. F., Nat. Med., 6, 470-473 (2000).

11) Robison L. M., Sclar D. A., Skaer T. L., Galin R. S., Clin. Rediatr. (Philadelphia), 38, 209-217 (1999).

12) Pliszka S. R., CNS Spectr., 8, 253-258 (2003).

13) Bymaster F. P., Katner J. S., Nelson D. L., Hemrick-Luecke S. K., Threlkeld P. G., Heiligenstein J. H., Morin S. M., Gehlert D. R., Perry K. W., Neuropsychopharmacology, 27, 699-711 (2002).

14) Morón J. A., Brockington A., Wise R. A., Rocha B. A., Hope B. T., J. Neurosci., 22, 389-395 (2002).

15) Giros B., Wang Y. M., Suter S., McLeskey S. B., Pifl C., Caron M. G., J. Biol. Chem., 269, 15985-15988 (1994).

16) Eshleman A. J., Carmolli M., Cumbay M., Martens C. R., Neve K. A., Janowsky A., J. Pharmacol. Exp. Ther., 289, 877—885 (1999).

17) Saito H., Murai S., Abe E., Masuda Y., Itoh T., Pharmacol. Biochem.
Behav. 42, 351-356 (1992).

18) Glowinski J., Iversen L. L., J. Neurochem., 13, 655-669 (1966).

19) Kurata K., Kurachi M., Hasegawa M., Kido H., Yamaguchi N., Jpn. J. Psychiatry Neurol., 41, 291-300 (1987).

20) Takeda H., Matsumiya T., Shibuya T., J. Chromatogr., 515, 265-278 (1990).

21) Brock J. W., Hamdi A., Ross K., Payne S., Prasad C., Pharmacol. Biochem. Behav., 52, 43-48 (1995).

22) Brock J. W., Farooqui S. M., Ross K. D., Payne. S., Prasad C., Physiol. Behav., 55, 997-1003 (1994).

23) Berridge C. W., Waterhouse B. D., Brain Res. Brain Res. Rev., 42, $33-84$ (2003).

24) Mallick B. N., Fahringer H. M., Wu M. F., Siegel J. M., Brain Res., 552, 333-337 (1991).

25) Siegel J. M., Rogawski M. A., Brain Res., 472, 213-233 (1988).

26) Porkka-Heiskanen T., Smith S. E., Taira T., Urban J. H., Levine J. E., Turek F. W., Stenberg D., Am. J. Physiol., 268, R1456-R1463 (1995).

27) Pujol J. F., Mouret J., Jouvet M., Glowinski J., Science, 159, 112-114 (1968).

28) Schildkraut J. J., Hartmann E., Psychopharmacologia, 27, 17-27 (1972).

29) Sinha A. K., Ciaranello R. D., Dement W. C., Barchas J. D., J. Neurochem., 20, 1289-1290 (1973).

30) Basheer R., Magner M., McCarley R. W., Shiromani P. J., Brain Res. Mol. Brain Res., 57, 235-240 (1998).

31) Gilad G. M., Rabey J. M., Gilad V. H., Life Sci., 40, 2401-2408 (1987).

32) Iuvone P. M., Morasco J., Dunn A. J., Brain Res., 120, 571—576 (1977).

33) Pycock C. J., Kerwin R. W., Carter C. J., Nature (London), 286, 74 76 (1980)

34) Leccese A. P., Lyness W. H., Neuropharmacology, 26, 1303-1308 (1987).

35) Haroutunian V., Knott P., Davis K. L., Psychopharmacol. Bull., 24, 341-344 (1988).

36) Deutch A. Y., Clark W. A., Roth R. H., Brain Res., 521, 311-315 (1990).

37) Rosin D. L., Clark W. A., Goldstein M., Roth R. H., Deutch A. Y., Neuroscience, 48, 831-839 (1992).

38) Swanson C. J., Perry K. W., Koch-Krueger S., Katner J., Svensson K. A., Bymaster F. P., Neuropharmacology, 50, 755-760 (2006).

39) Mateo Y., Pineda J., Meana J. J., J. Neurochem., 71, 790-798 (1998).

40) Lategan A. J., Marien M. R., Colpaert F. C., Brain Res., 523, 134 138 (1990).

41) Lategan A. J., Marien M. R., Colpaert F. C., Life Sci., 50, 995-999 (1992).

42) Asakura W., Matsumoto K., Ohta H., Watanabe H., Pharmacol. Biochem. Behav., 46, 111-115 (1993). 